\title{
Analysis of the genetic predisposition to develop a Myocardial Infarction in a sample of Moroccan patients.
}

Fatima Ezzahra Anaibar 1, Sana Essenhagi 3, Said Nassor Abeid 1, Hamid Farhane', Majida Motrane ${ }^{1}$, Rachida Habbal $^{2}$, Norredine Ghalim ${ }^{3}$ \& Nourdin Harich ${ }^{1}$

${ }^{1}$ Team of Anthropogenetic Sciences and Biotechnologies - Faculty of Sciences - Chouaib Doukkali University, El

Jadida, Morocco.

${ }^{2}$ CHU IBN ROCHD Cardiology Center, Casablanca, Morocco

${ }^{3}$ Biochemistry laboratory at the Institut Pasteur Casablanca, Morocco

\begin{abstract}
Myocardial infarction (MI) is a multifactorial coronary artery disease influenced by environmental and genetic factors. Being one of the leading causes of morbidity and mortality in developed countries, it is becoming a national health concern in developing countries such as Morocco. In the present work, we aim to study the association between 4 Alu polymorphisms (ACE, FXIII-B, TPA-25 and APOA1) genes and the risk of MI in the Moroccan population.

210 patients with a history of myocardial infarction and 203 healthy individuals were included in this study. The Alu polymorphisms were determined by the PCR technique followed by direct electrophoresis.

Only the I / I_APOA1 genotype showed a significant association with the risk of MI $(\mathrm{p}<0.001 ; \mathrm{OR}=2.171)$, while genotypes carrying the $\mathrm{D}$ allele $(\mathrm{D} / \mathrm{D}$ or $\mathrm{I} / \mathrm{D})$ showed a protective effect $(\mathrm{p}<0.001$; $\mathrm{OR}=0.46)$. Patients presenting an association between I / I and a high level of triglycerides $(p=0.020, O R=2.14)$ ), as well as a significant association with a high level of LDLs $(\mathrm{p}=0.035, \mathrm{OR}=2,00)$. Subjects carrying the I / I_APOA1 genotype with dyslipidemia or hyperglycemia are more likely to develop MI, as are those carrying I / D_APOA1 with hyperglycemia or high cholesterol.
\end{abstract}

Keywords: Myocardial infarction, Polymorphism, Alu ApoA1, Hyperglycemia, Dyslipidemia

\section{INTRODUCTION:}

Cardiovascular disease (CVD) and particularly myocardial infarction (MI), is the leading cause of death in the world [1]. MI is defined as a necrosis of the heart muscle at the level of the myocardium, the MI occurs when one of the coronary arteries is occluded for a long time and causes destruction of the heart muscle. The risk of developing an MI is further increased when complication factors are associated [2]. Morbidity and coronary mortality are associated with an increase of levels in LDL cholesterol and in triglycerides (TG), and also with a decrease in HDL cholesterol levels [3].

In order to monitor the health of our population, we have studied the association between 4 Alu polymorphisms located on the ACE, FXIII-B, TPA25 and APOA1 genes and the risk of MI in a sample of the Moroccan patients, using Alu family markers to study the genetic structure of our samples, due to their unique mutation mechanism [4-6].

The aim of the present study is to examine whether these four common Alu insertion/deletion polymorphisms are associated with the risk of myocardial infarction by comparing results of 2 groups (patients and controls).

\section{SUBJECTS AND METHODS:}

In the present study, a sample of 413 unrelated subjects were enrolled: 210 patients with a history of myocardial infarction recruited from the cardiology service of Ibn Rochd University Hospital, Casablanca / Morocco, and a control group consisted of 203 healthy individuals. Clinical, biochemical and anthropometric data were recorded for all participants in a well-established individual questionnaire. The blood sampling was carried out in accordance with the recommendations of the Human Genome Project and the ethical rules in force in Morocco and have also received the approval of the Ethics Committee of the Institut Pasteur.

The polymorphism analysis of the Alu insertions located in four different autosomal genes, suspected to be in association with susceptibility to MI (APOA1, FXIII-B, ACE and PLAT TPA25) was performed on the two samples. Genomic DNA was extracted from the leukocyte fraction of whole blood samples, using the Wizard Genomic DNA Purification Kit according to the manufacturer's instructions (Promega Corporation, Madison, WI, USA). The PCR conditions are described in table 1, and its products were directly separated on $2 \%$ agarose gel. 
Table 1. PCR conditions of the markers (ACE, PLAT TPA-25, Alu FXIII-B and APOA1).

\begin{tabular}{|c|c|c|c|}
\hline & Primers sequences & PCR products & References \\
\hline$A l u A C E$ & $\begin{array}{l}\text { 5'CTGGAGACCACTCCCATCCTTTCT3' } \\
\text { 5'GATGTGGCCATCACATTCGTCAGAT3' }\end{array}$ & $\begin{array}{l}490 \mathrm{pb} \\
190 \mathrm{pb}\end{array}$ & Tiret et al.(1992) [7] \\
\hline PLAT TPA-25 & $\begin{array}{l}\text { 5' GTG AAA AGC AAG GTC TAC CAG 3' } \\
\text { 5' GAC ACC GAG TTC ATC TTG AC- 3' }\end{array}$ & $\begin{array}{l}570 \mathrm{pb} \\
260 \mathrm{pb}\end{array}$ & Tiret et al. (1996) [7] \\
\hline AluFXIII-B & $\begin{array}{l}\text { 5'TCAACTCCATGAGATTTTCAGAAGT3' } \\
\text { 5'CTGGAAAAAATGTATTCAGGTGAGT3 }\end{array}$ & $\begin{array}{l}700 \mathrm{pb} \\
410 \mathrm{pb}\end{array}$ & Batzer et al.(1996) [8] \\
\hline ApoA1 & $\begin{array}{l}\text { 5'AAGTGCTGTAGGCCATTTAGATTAG-3' } \\
\text { 5'AGTCTTCGATGACAGCGTATACAGA-3' }\end{array}$ & $\begin{array}{l}510 \mathrm{pb} \\
210 \mathrm{pb}\end{array}$ & Batzer et al.(1994) [8] \\
\hline
\end{tabular}

Allelic frequencies of the markers were estimated using Biosys1 computer program [9]. Mean comparisons, odds ratios and their respective p-values and 95\% confidence intervals (95\% CI) were calculated using SPSS (IBM SPSS Statistics) [10].

\section{Results and discussion:}

1.1 Association of genotypes with anthropometric parameters:

Among the four polymorphisms analyzed, we did not find a significant association between the Ins / Del genotypes of three Alu insertions at the FXIII$\mathrm{B}, \mathrm{ACE}$ and TPA25 and IM in the complete sample and also by gender. Concerning body mass index, no significant association was found between the genotypes of the three systems and the different BMI classes.

Nevertheless, our results showed no significant association between the APOA1 gene genotypes and gender. Regarding the Body Mass Index (BMI), the analysis showed a significant association between MI patients that carried Ins/ Ins genotype of APOAI and BMI $(\mathrm{p}=0.026$; OR = $2.589 ; 95 \%$ CI [1.116-5.598]), when comparing the overweight subgroup with the normal weight subgroup. Indeed, the value of the odds ratio obtained (2.589), would indicate that overweighted individuals carrying Ins/ Ins genotype have a multiplicated risk by about 2.6. Obesity being a major risk factor for reaching cardiovascular disease and therefore also MI (figure1).

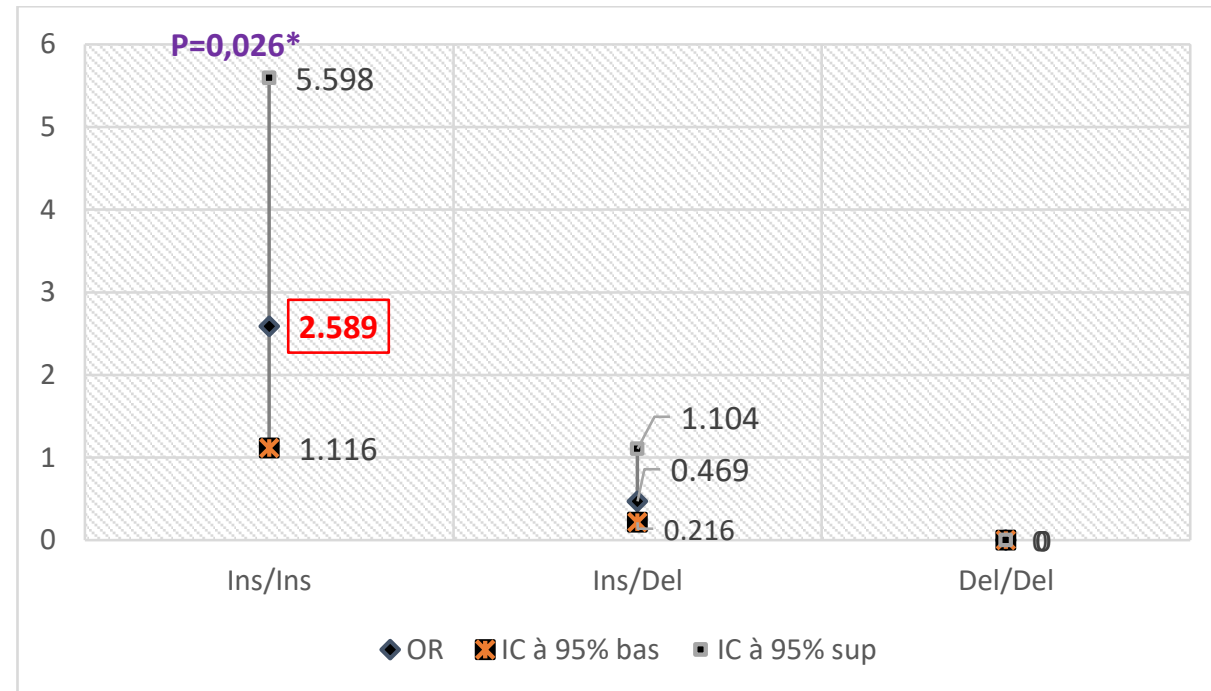

Fig. 1. Association of APOA1 genotypes with BMI.

1.2 Association of genotypes with biochemical parameters:
Regarding the Ins / Del genotypes of Alu polymorphisms at the FXIII-B, ACE and TPA25 genes, the statistical analysis did not shown any 
significant difference in biochemical parameters between patients and controls.

Regarding APOAI Alu-polymorphism, our results show that MI patients carrying Ins/ Ins genotype frequently present an hyperglycemic profile compared to other genotypes ( $\mathrm{p}=0.004$, OR $=3.366 ; 95 \%$ CI [1.466-7.727]), suggesting an association of the Ins / Ins genotype with hyperglycemia in patients with MI. The odds ratio found indicates that they have a 3.366-times higher risk of developing diabetes, something that might probably predispose them to develop MI or heart conditions that leed to it. On the other hand, our analysis showed that the APOAI * Ins / Del genotype seems to be a protective genotype against diabetes, $(\mathrm{p}=0.007, \mathrm{OR}=0.303 ; 95 \%$ CI $[0.126$ 0.727])(Figure 2).

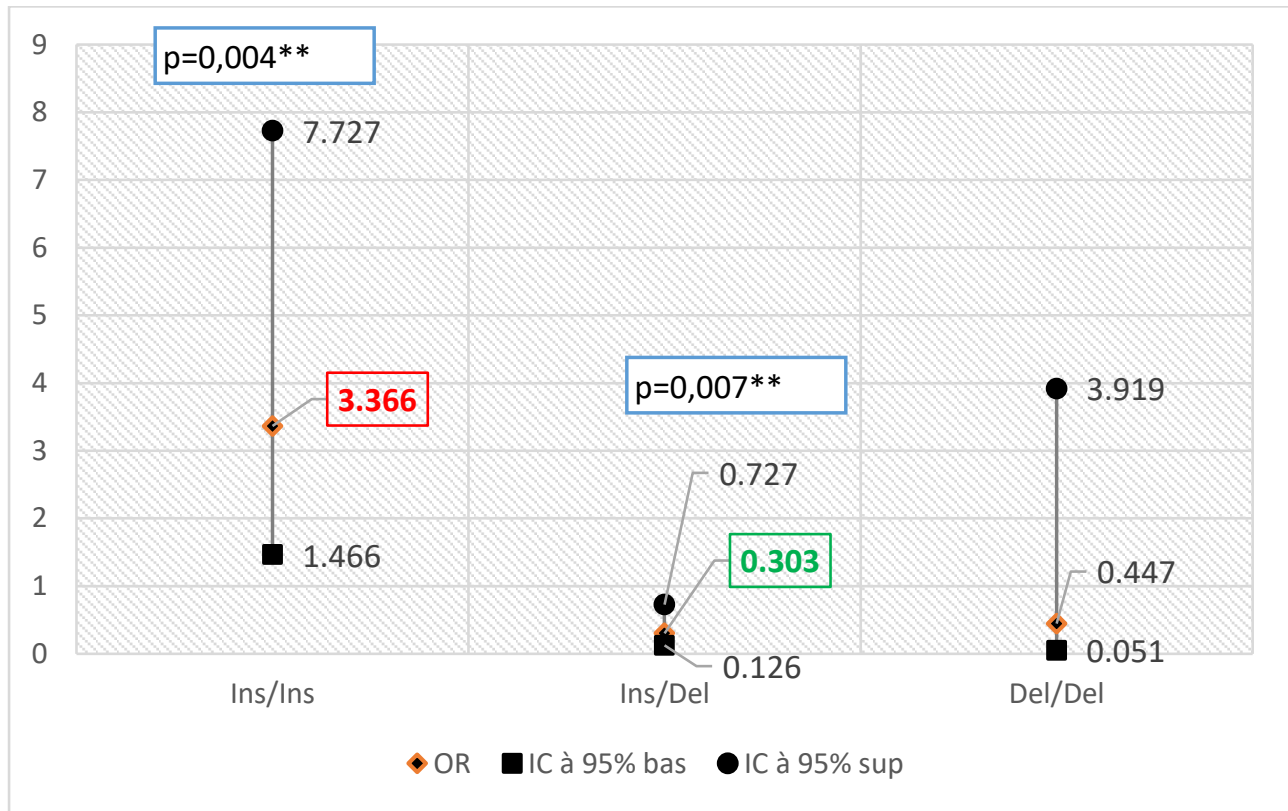

Fig.2. Association of APOA1 genotypes with glycemia.

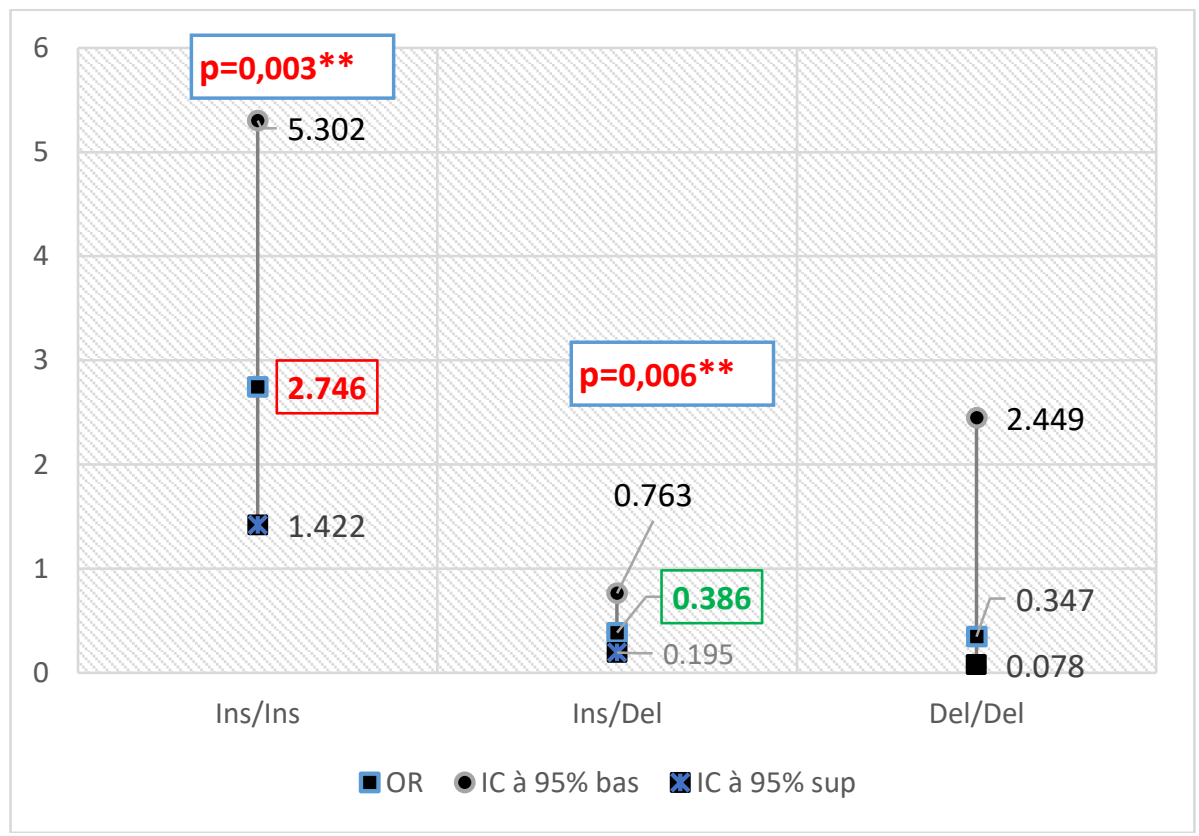

Fig. 3. Association of APOA1 genotypes with cholesterol levels.

Compared to hypercholesterolemia, the analysis of the association of Alu-APOAI genotypes with cholesterol showed that the Ins / Ins genotype is positively associated with hypercholesterolemia ( $p$
$=0.003, \mathrm{OR}=2.746 ; \mathrm{IC} 95 \%[1.422-5.302])$, and that the Ins / Del genotype is protective against hypercholesterolemia $(\mathrm{p}=0.006, \mathrm{OR}=0.386 ; 95 \%$ CI [0.195-0.763]). We showed also, that carriers of 
Ins / Ins genotypes have a 2.746-times higher risk of becoming hypercholesterolemic compared to carriers of other genotypes and that carriers of the Ins / Del genotype seems to be protected against increased cholesterol levels in the blood (Figure 3).

It was also noted that the Ins/ Ins genotype in MI patients is associated with a high level of triglycerides $(p=0.020$, OR $=2.141 ; 95 \%$ CI [1.125-4.073]); the odds ratio value suggests the predisposing effect of the Ins / Ins genotype to hypertriglyceridemia, with a risk multiplied by 2.141 times. Similarly, patients with an Ins / Ins genotype present a significant association with higher LDL levels $(>2 \mathrm{~g} / \mathrm{L}$ ), and the odds ratio value shows that the risk of being hyper-LDL is multiplied by approximately 2 times $(\mathrm{p}=0.035$, OR $=2.000 ; 95 \%$ CI [1.050-3.810])(Figure 4).
In the light of these last results, it seems that Ins / Ins genotype plays a very important role in the lipid disorders like hypercholesteremia, triglyceridemic and high LDL levels, which increases the risk of developing a myocardial infarction. Conversely, the work carried out by Frossard et al. (1986), demonstrated that the Del allele had a high frequency in patients with atherosclerosis compared to controls[11], while the study conducted by Lim et al. (1986) demonstrated an association of the Del allele with low levels of the good cholesterol (HDL)[12]. In relation to hypertension, the Ins / Ins genotype of Alu-APOAI was also found to be associated $(\mathrm{p}=0.035, \mathrm{OR}=$ $2.056 ; 95 \%$ CI [1.053-4.014]). Carriers of this genotype would also have a doubled risk of becoming hypertensive and therefore of developing MI.

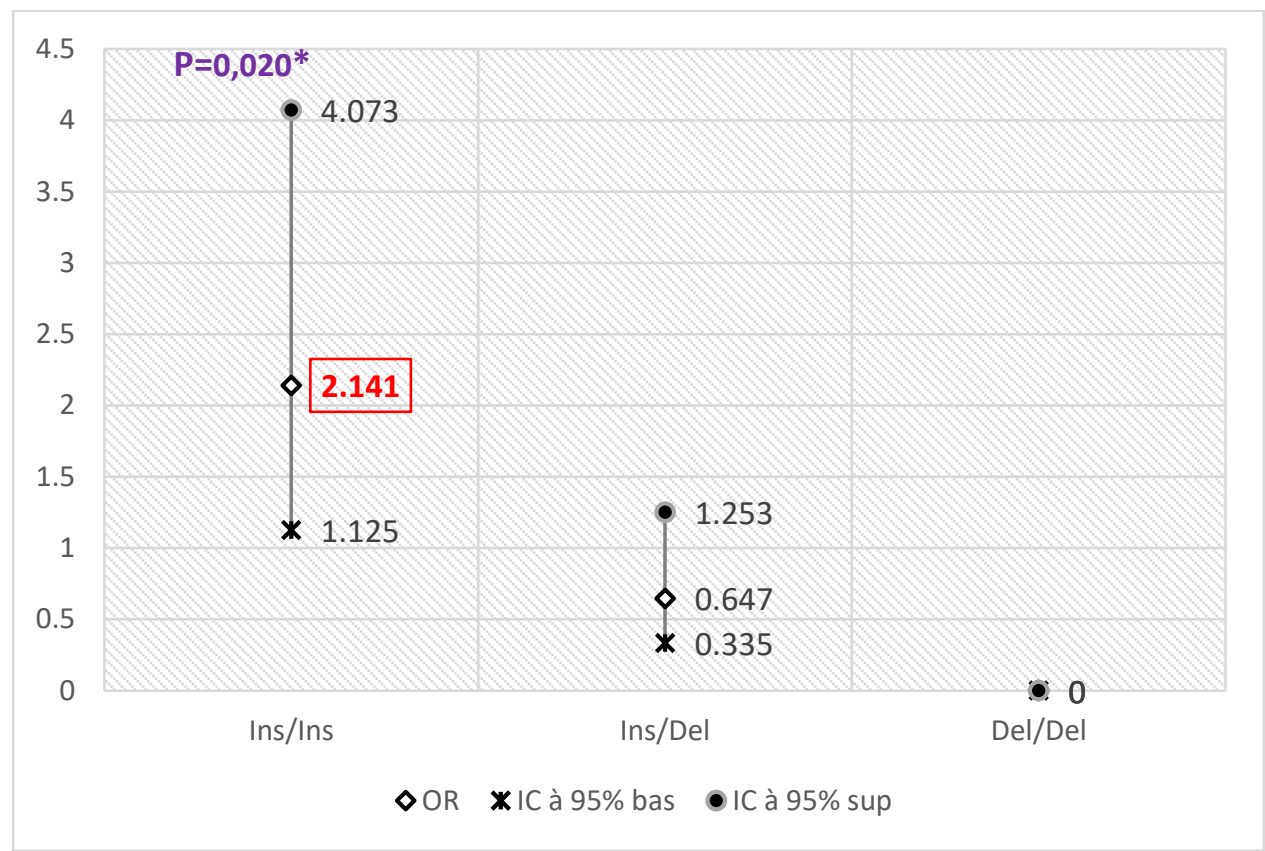

Fig.4. Association of APOA1 genotypes with triglycerides levels.

\subsection{Multivariate associations of studied Alu with the risk of $\mathrm{Ml}$}

Except for FXIII-B Alu in MI patient's group, all the genotype and allele frequency distributions of the four polymorphisms followed Hardy-Weinberg equilibrium ( $\mathrm{p}>0.05)$.

The most interesting results were recorded in association with the APOA1 polymorphism (Figure 5). The distribution of the Alu-APOAI genotypes shows that there is a significant association between the Ins / Ins genotype and a high risk of myocardial infarction $(\mathrm{p}<0.001 ; \mathrm{OR}=2.171,95 \% \mathrm{CI}=[1.547$ $3.049]$ ), due to the protective effect exerted by the Del allele $\mathrm{p}<0.001 ; \mathrm{OR}=0.46 ; 95 \% \mathrm{CI}=[0.323$ $0.655]$ ), and by genotypes carrying Del (Del / Del and Ins / Del). Our results are in line with several studies that have shown the involvement of the APOAI gene and its various polymorphisms in the susceptibility of heart disease through its associations with high levels of triglycerides and low HDL-C[12, 13].

No significant difference was observed when comparing MI patients with healthy controls for Alu insertion at the FXIII-B and TPA genes. Likewise, a study on the French population showed that there is no significant difference between allelic frequencies of Alu-TPA25 encountered in cardiac patients and in controls [14].

Another genetic study showed that the Ins / Del polymorphism of TPA25 did not impact the levels and activity of the tissue plasminogen activator enzyme and that Ins/ Ins and Ins/ Del genotypes 
were common in subjects who have suffered a nonfatal myocardial infarction[15]. In contrast, the work of Jern et al. (1999) demonstrated a significant association of the Ins / Ins genotype and the rate of production of the tissue plasminogen activating enzyme[16].

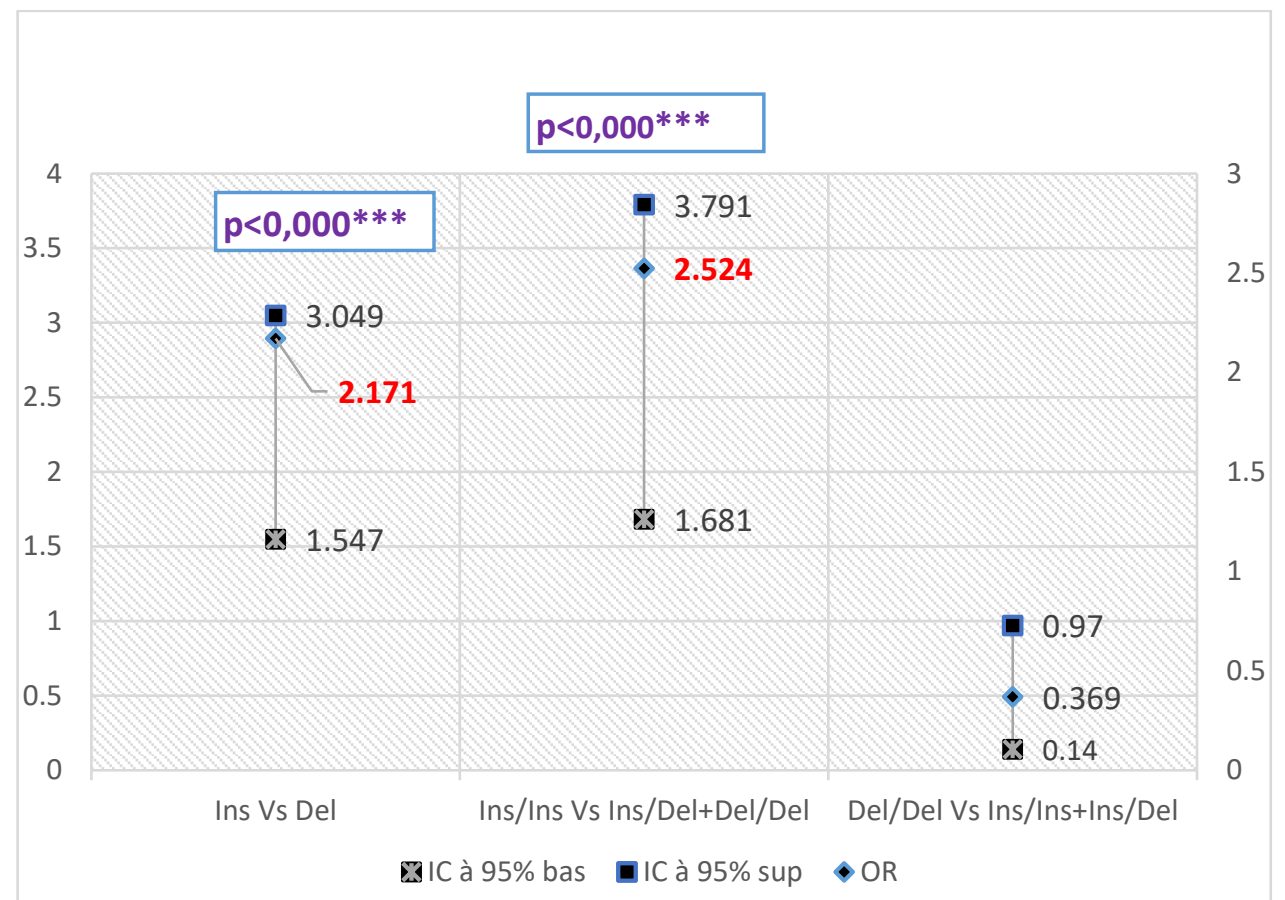

Fig.5. Distribution of allele and genotypic frequencies in the groups studied and analysis of the association with MI.

In the same way, statistical analysis did not show an association between the Ins/ Del polymorphism of the ACE gene and the risk of myocardial infarction in our cohort. These results were confirmed by Larsen et al. (2000), who had shown that it can only affect the plasma activity of ACE without affecting blood pressure [17].

The association between the ACE Alupolymorphism and the risk of myocardial infarction (MI) has been extensively studied. A meta-analysis by Chen et al. (2013) showed that the Del allele represents a possible risk factor for the incidence of myocardial infarction worldwide [18]. It has also been reported that the ACE Ins/ Del polymorphism could influence the right ventricle and the performance of the myocardium in patients with myocardial infarction [19], can have an effect on improving the susceptibility to cardiovascular risk in patients with type 2 diabetes [20].

Other studies have highlighted that the Del/ Del genotype is an important risk factor for myocardial infarction [21], another one conducted by (Hooper et al., 2002) found that this genotype of ACE is associated with myocardial infarction in men and not associated in women [22].

\section{CONCLUSION:}

To determine the association between genetic factors and the incidence of cardiovascular diseases especially the MI in the Moroccan population, we have analyzed the correspondence between four Alu polymorphisms located at the ACE, FXIII-B, TPA and APOA1 genes and the risk of MI.

Only APOAI-Alu genotypes distribution shows that there is a significant predisposition of Ins/ Ins genotype to develop a myocardial infarction with a high risk, and that there is some protective effect exerted by the Del allele, and by the genotypes carriers of Del (Del/ Del and Ins/ Del). These results were confirmed by comparing the allelic distributions between MI patients and controls. On the other hand, our results suggest that subjects carrying the Ins/ Ins genotype of APOAI and having at the same time high LDL cholesterol levels, hyperglycemia and hypertriglyceridemia, as well as patients carrying Ins/ Del APOAI genotype together with hyperglycemia and hypercholesterolemia may be more predisposed to develop myocardial infarction. Finally, we can consider that the I/D Alu-APOA1 polymorphism could constitute a good genetic marker for the diagnosis of the susceptibility to develop MI in the Moroccan population.

\section{Reference:}

1. H.Zhang, et al., Hirudin protects against isoproternol-induced myocardial infraction by alleviating oxidative via an Nrf2 dependent manner, in International journal of biological macromolecules, 162: p. 425-435, (2020) 
2. J.Daccache, et al, Les Canadiens partiellement Français sont susceptibles à une augmentation des facteurs de risque cardiovasculaire: une étude de cohorte rétrospective basée sur la population, in Annales de Cardiologie et d'Angéiologie, Elsevier, (2020)

3. P.Libby, What have we learned about the biology of atherosclerosis? The role of inflammation, in The American journal of cardiology, 88(7): p. 3-6, (2001)

4. M.A.Batzer, and P.L. Deininger, Alu repeats and human genomic diversity, in Nature reviews genetics, 3(5): p. 370-379, (2002)

5. W.S.Watkins, et al., Genetic variation among world populations: inferences from 100 Alu insertion polymorphisms, in Genome research, 13(7): p. 1607-1618, (2003)

6. S.N.Abeid, et al., Human population genetics of Comoros islanders: Alu polymorphisms and the peopling of the three major islands, in Gene Reports, 22: p. 100927, (2021)

7. L.Tiret, et al., Evidence, from combined segregation and linkage analysis, that a variant of the angiotensin I-converting enzyme (ACE) gene controls plasma ACE levels, in American journal of human genetics, 51(1): p. 197, (1992)

8. M.A.Batzer, et al., Genetic variation of recent Alu insertions in human population, in Journal of molecular evolution, 42(1): $p$. 22-29, (1996)

9. D.Swofford, and R. Selander, BIOSYS-1: a computer program for the analysis of allelic variation, in population genetics and biochemical systematics, (1989)

10. Spss, I., IBM SPSS Statistics for Windows, version 25, in Armonk, NY: IBM SPSS Corp,[Google Scholar],( 2017)

11. P.Frossard, et al., Genetic markers for coronary atherosclerosis in the human apolipoprotein AI-CIII-AIV gene complex, in Am J Hum Genet, 39(Suppl 589), (1986)

12. D.Lim, et al., Deletion of an Alu sequence in the 5' of the apolipoprotein AI gene associated with decreased serum HDLcholesterol levels, in Am J Hum Genet, 39: p. 621, (1986)

13. J.L.Breslow, Transgenic mouse models of lipoprotein metabolism and atherosclerosis, in Proceedings of the
National Academy of Sciences, 90(18): p. 8314-8318, (1993)

14. A.Falchi, et al., Prevalence of genetic risk factors for coronary artery disease in Corsica island (France), in Experimental and molecular pathology, 79(3): p. 210213, ( 2005)

15. J.G.Van der Bom, et al., Tissue plasminogen activator and risk of myocardial infarction: the Rotterdam Study, in Circulation, 95(12): p. 26232627, (1997)

16. C.Jern, et al., Gene polymorphism of $t-P A$ is associated with forearm vascular release rate of $t-P A$, in Arteriosclerosis, thrombosis, and vascular biology, 19(2): p. 454-459, ( 1999)

17. B.Agerholm-Larsen, B.G. Nordestgaard, and A. Tybjærg-Hansen, ACE gene polymorphism in cardiovascular disease: meta-analyses of small and large studies in whites, in Arteriosclerosis, thrombosis, and vascular biology, 20(2): p. 484-492, (2000)

18. Y.Chen, et al., Angiotensin-converting enzyme insertion/deletion polymorphism and risk of myocardial infarction in an updated meta-analysis based on 34993 participants, in Gene,522(2): p. 196-205, (2013)

19. O.Ozturk, et al., Influence of angiotensinconverting enzyme I/D gene polymorphism on the right ventricular myocardial performance index in patients with a first acute anterior myocardial infarction, in Circulation Journal, 69(2): p. 211-215, (2005)

20. M.Ganesan, et al., The relationship of $A C E$ and CETP gene polymorphisms with cardiovascular disease in a cohort of Asian Indian patients with and those without type 2 diabetes, in Journal of Diabetes and its Complications, 25(5): p. 303-308, (2011)

21. S.Mehri, et al., Angiotensinogen gene polymorphism in acute myocardial infarction patients, in Journal of the Renin-Angiotensin-Aldosterone System, 12(1): p. 42-47, (2011)

22. W.C.Hooper, et al., Relationship of venous thromboembolism and myocardial infarction with the renin-angiotensin system in African-Americans, in American journal of hematology, 70(1): p. 1-8, (2002) 